\title{
ANTECEDENTES EXITOSOS PROPIOS - VALOR AÑADIDO PARA LA EDUCACIÓN SUPERIOR EN COLOMBIA
}

\author{
Edgardo Sánchez \\ Universidad de la Costa (Colombia) \\ esanchez2@cuc.edu.co \\ Marcial Conde \\ Universidad de la Costa (Colombia) \\ Freddy Marín \\ Universidad de la Costa (Colombia) \\ Alicia Inciarte \\ Universidad de la Costa (Colombia) \\ Jesús N. García \\ Universidad de León/Universidad de la Costa \\ Judit García-Martín \\ Universidad de Salamanca
}

Fecha de Recepción: 2 Abril 2019

Fecha de Admisión: 30 Abril 2019

\section{RESUMEN}

En los últimos años se han venido ensayando estrategias exitosas diversas, tanto desde el contexto propio de la universidad, como desde otros contextos internacionales. Estos antecedentes empíricos, apoyados por datos impresionísticos o validados con evidencias internacionales justifican la formalización de un programa de investigación e innovación docente universitaria centrado en las competencias genéricas de lectura crítica LC y construcción textual CT. Para ello, parece pertinente el analizar estos antecedentes, primero del propio contexto y después los procedentes de contextos externos e internacionales. En el reciente autoinforme para la acreditación de la institución universitaria ante el Ministerio de Educación Nacional se identificaron diferentes estrategias exitosas, cuya lógica y forma de implementación merece la pena revisar para el desarrollo del tema que nos ocupa. Por una parte, se vienen promoviendo estrategias como el uso de rúbricas, el acompañamiento de actividades, sugiriéndose el fomento de metodologías activas como el ABP, el MC, o el eLearning, sea a través de MOOCs u otros medios. En primer lugar, conviene revisar las estrategias utilizadas para el factor 2, estudiantes, sobre todo en lo que hace a la permanencia; y las estrategias para paliar la deserción y que se amplía en el factor 9 de bienestar institucional. En segundo lugar, las estrategias implementadas para el factor 3 profesorado, parecen muy relevantes para esta inves- 


\section{ANTECEDENTES EXITOSOS PROPIOS - VALOR AÑADIDO PARA LA EDUCACIÓN SUPERIOR EN COLOMBIA}

tigación. Conviene revisar las estrategias implementadas con éxito de buenas prácticas docentes, capacitación docente en docencia inicial, plan de acompañamiento docente (PAD). En tercer lugar, la revisión de las actuaciones y estrategias utilizadas en el factor 8 de autoevaluación y autorregulación son de gran interés. Se revisan, también, otras experiencias exitosas de gran interés. "Proyecto de Investigación Competitivo CONV-ÍNDEX Núm. 13-2018, Código Proyecto INV. 150-01007-13 Universidad de la Costa (Colombia) - Universidad de León (España). Evaluado por la ACAC (MEN Colombia)"

Palabras clave: estrategias instruccionales exitosas; metodologías activas; permanencia y abandono estudiantil; plan acompañamiento docente

\section{ABSTRACT}

Succesful antedents own - value added for higer education in Colombia. In recent years, various successful strategies have been tried, from the context of the university itself, as well as from other international contexts. These empirical backgrounds, supported by impressionistic data or validated with international evidence, justify the formalization of a university teaching research and innovation program focused on the generic competences of critical reading LC and textual construction CT. For this, it seems pertinent to analyze these antecedents, first of the context itself and then those coming from external and international contexts. In the recent self-report for the accreditation of the university institution before the Ministry of National Education, different successful strategies were identified, whose logic and manner of implementation is worth reviewing for the development of the subject that concerns us. On the one hand, strategies such as the use of rubrics, the accompaniment of activities, are being promoted, suggesting the promotion of active methodologies such as ABP, MC, or eLearning, whether through MOOCs or other means. In the first place, it is convenient to review the strategies used for factor 2, students, especially in terms of permanence; and the strategies to alleviate the desertion and that is extended in the factor 9 of institutional well-being. Second, the strategies implemented for teacher factor 3 seem very relevant for this research. It is convenient to review the strategies successfully implemented for good teaching practices, teacher training in initial teaching, teacher support plan (PAD). Third, the review of the actions and strategies used in factor 8 of self-evaluation and self-assessment are of great interest. Other successful experiences of great interest are also reviewed. "Competitive Research Project CONVÍNDEX No. 13-2018, Project Code INV. 150-01-007-13 University of the Coast (Colombia) University of León (Spain). Evaluated by the ACAC (MEN Colombia)"

Keywords: successful instructional strategies; active methodologies; student permanence and abandonment; teacher support plan

\section{ANTECEDENTES EXITOSOS (UNIVERSIDAD DE LA COSTA)}

En los últimos años se han venido ensayando estrategias exitosas diversas, tanto desde el contexto propio de la universidad, como desde otros contextos internacionales. Estos antecedentes empíricos, apoyados por datos impresionísticos o validados con evidencias internacionales justifican la formalización de un programa de investigación e innovación docente universitaria centrado en las competencias genéricas de lectura crítica LC y construcción textual CT. Para ello, parece pertinente el analizar estos antecedentes, primero del propio contexto y después los procedentes de contextos externos e internacionales.

En el reciente autoinforme para la acreditación de la institución universitaria ante el Ministerio de Educación Nacional (MEN) se identificaron diferentes estrategias exitosas, cuya lógica y forma de implementación merece la pena revisar para el desarrollo del tema que nos ocupa. Por una parte, se vienen promoviendo estrategias como el uso de rúbricas, el acompañamiento de actividades 
(CUC-AIA, 2018), sugiriéndose el fomento de metodologías activas como el ABP, el MC, o el eLearning, sea a través de MOOCs u otros medios.

\section{Factor estudiantes}

En primer lugar, conviene revisar las estrategias utilizadas para el factor 2, estudiantes (CUCAIA, 2018, pp. 90-132), sobre todo en lo que hace a la permanencia (CUC-AIA, 2018, pp., 97 y ss.; pp., 106 y ss.); y las estrategias para paliar la deserción (CUC-AIA, 2018, pp., 112 y ss.) y que se amplía en el factor 9 de bienestar institucional (CUC-AIA, 2018, pp., 799 y ss.). Por una parte, desde el Plan Educativo Institucional (PEI) se determina la cultura de permanencia, lo que llevó al desarrollo del Programa de Acompañamiento y Seguimiento para la Permanencia Estudiantil (PASPE). Por otra parte, el análisis de las tasas de graduación, y el uso del software SPADIES (Sistema para la Prevención de la Deserción de la Educación Superior) como sistema que permite hacer el seguimiento de las cifras de deserción. Por lo tanto, son focos relevantes para revisar y que sirven de antecedentes exitosos claves, el problema de la permanencia (CUC-AIA, pp., 106 y ss) y la deserción estudiantil (CUC-AIA, 107 y ss).

El análisis de seguimiento de tres años de los factores determinantes de la deserción temprana identificó motivaciones en variables multifactoriales de gran interés y relevancia para la toma de decisiones de mejora (Fragozo Torres, CUC1, 2009). El menor status socioeconomico lo refieren el $53 \%$ de los estudiantes, como tener personas a su cargo, estar trabajando, la ocupación de los padres o contraer obligaciones económicas. Además, los factores personales se dan en el $25 \%$ de los casos, como convivir o depender de los padres, tener hijos, procedencia. Seguidamente, los factores académicos lo indican el 17\%, relativos a inadecuada orientación o menor rendimiento. Y por último, los factores institucionales solo afectan al $5 \%$ de los estudiantes, relativos a dificultades con las metodologías docentes. Junto a ese estudio, otro de seguimiento de otros tres años, permitió la identificación de motivaciones de la deserción tardía (CUC2, 2013) resaltando dos tipos de variables multifactoriales, en torno al factor socioeconómico y a factores de personalidad. El hecho de que la extracción social de los estudiantes de la CUC lo sean de los estratos 1-3 casa con los datos de estos estudios sobre deserción y con el hecho de que obtengan resultados menores en las pruebas de competencias genéricas (SaberPro), cuestiones abordadas desde diferentes estrategias y que deben ser consideradas en la planificación del presente programa de investigaciones (CUC-AIA, 2018; CUC-Departamento de Estadística, 2017a; 2017b).

En este sentido se han implementado diferentes estrategias imaginativas para paliar la deserción estudiantil. Interesa analizar para este programa de investigación, sobre todo, las de aprender a aprender, refuerza-T y bienestar en la clase (CUC-AIA, 2018, pp., 112-119), si bien se vienen desarrollando eficazmente otras estrategias muy relevantes para el problema de la deserción.

\section{Factor profesorado}

En segundo lugar, las estrategias implementadas para el factor 3 profesorado, parecen muy relevantes para esta investigación (CUC-AIA, 2018, pp., 177-181). Conviene revisar las estrategias implementadas con éxito de buenas prácticas docentes, capacitación docente en docencia inicial, plan de acompañamiento docente (PAD). Siendo las de mayor interés el explicitar las mejores prácticas o prácticas basadas en la evidencia empírica o científica y revisar lo que se ha validado como eficaz y eficiente a nivel internacional (García et al., 2016).

\section{Factor de autoevaluación y autorregulación}

En tercer lugar, la revisión de las actuaciones y estrategias utilizadas en el factor 8 de autoevaIuación y autorregulación son de gran interés. En concreto, el programa implementado desde el 


\section{ANTECEDENTES EXITOSOS PROPIOS - VALOR AÑADIDO PARA LA EDUCACIÓN SUPERIOR EN COLOMBIA}

Centro de Excelencia Docente, "Saber te lleva lejos" ha desarrollado estrategias diversas de promoción de las competencias genéricas incidiendo en los sistemas de evaluación. Han resultado exitosas las estrategias de (i) formación y acompañamiento a directivos y profesores mediante actuaciones transversales en las materias; (ii) prueba diagnóstica de competencias genéricas a estudiantes de nuevo ingreso; (iii) valoración de competencias genéricas y específicas en estudiantes de S3, S5 y S7; y (iv) diseño de pruebas por departamentos académicos (CUC-AIA,2018, pp., 769-770). Todas ellas relevantes para esta investigación y permiten plantear varias cuestiones nucleares para el presente programa de investigación. Por una parte, las actuaciones transversales curriculares en las materias de mejora de competencias genéricas sugieren indagar sobre las acciones desarrolladas; la evaluación de su impacto; el seguimiento por los profesores; las diferencias en resultados de Ios alumnos entre los que tienen profesores que siguen las acciones y las que no; las áreas en que se han implementado. Y otras cuestiones cabría hacerse respecto a las pruebas diagnósticas. En concreto, sobre la disponibilidad de resultados y poder validar las pruebas; la existencia o no de algún patrón diferencial por áreas o campos; a cuántos estudiantes llega; y las estrategias desarrolladas de tipo transversal (ej., en LC, CT, posibilidad de algún MOOC).

Una ilustración de este tipo de aportaciones, son la identificación de los antecedentes del papel de variables psicoeducativas. La importancia de los patrones psicológicos y educativos en los resultados académicos es indudable. Se han utilizado múltiples constructos de gran relevancia para poder comprender los resultados de aprendizaje, académicos y niveles de grado y logro. Enunciemos algunos de ellos que podrán ser incluidos en el muestreo de la investigación: la motivación de logro (ej., atribuciones), la autoeficacia percibida, las actitudes, las características personales, los estilos de aprendizaje y de pensamiento, el uso de estrategias, factores de inteligencia emocional, los procesos de autorregulación, entre otros, lo que permite contrastar con muchos estudios internacionales recientes en el campo, incluyendo estudios internacionales de comparación con muestras colombianas (Cheng \& Lin, 2018; Cuesta et al., 2018; Díaz \& García, 2016; Fang et al., 2017; García-Martín \& García-Sánchez, 2017; Habok \& Magyar, 2017; Mnez-Ferrer et al., 2018; Nie et al., 2017; Oriol et al., 2018; Puente et al., 2018; Robledo \& García, 2018; Romera et al., 2018, Sinval et al., 2018; Widlund et al., 2017). En una revisión reciente, en que se revisan todas las intervenciones pedagógicas con enfoque en la autorregulación, implementadas en China durante las últimas dos décadas, de 603 estudios, sólo pudieron incluirse en los análisis 59, y de ellos se identifican solo cuatro estudios cuasi-experimentales, frente al resto de registro de experiencias ( $\mathrm{Li}, \mathrm{Ye}$, Tang, Zhou, Hu, 2018), lo que confirma el estado general a nivel, no solo de Colombia o de USA, sino generalizado, por lo que la justificación de esta investigación y con la metodología prevista, es una opción con potencial heurístico, de contribución teórica y aplicada.

\section{Focos de actuación e incidencia}

De los argumentos y evidencias anteriores, se puede sintetizar en diferentes focos las aportaciones de experiencias propias y la incidencia de sistematizar un programa de investigación de esta naturaleza, tanto para la institución superior propia, en donde se desarrollan, como para la comunidad nacional e internacional de instituciones de enseñanza superior.

Por una parte, el fomento de Investigación e Innovaciones Docentes, tanto propias como nacionales e internacionales, tanto en las áreas de humanidades y educación como en el resto de ámbitos de enseñanza superior. En segundo lugar, la diversificación de valoraciones de enseñanza y aprendizaje (Sistemas de Evaluación SSEV), que con foco en las competencias genéricas, es evidente que afectan a las competencias específicas. En tercer lugar, la mejora de las valoraciones de enseñanza y aprendizaje (Sistemas de Evaluación Docentes SSEV). En cuarto lugar, la promoción de 
Metodologías Activas e Innovadoras (MMAC), tan necesario y deseable en una sociedad en cambio vertiginoso en una sociedad de la información y la comunicación y en una web ubicua. En quinto lugar, la promoción de Competencias Generales, centradas sobre todo en las Comunicativas de Lectura Crítica y Construcción Textual. En sexto lugar, el incidir en resultados en Saber Pro (Culminación de Saber3, Saber5, Saber11), cuestión nuclear en la formación de profesionales egresados de las universidades, puesto que no basta con la formación específica, se trata de profesionales de alta especialización que han de dominar las competencias para ser ciudadanos ejemplares y fructíferos para la comunidad a la que sirven y para la comunidad internacional en un mundo global. Y en séptimo lugar, las experiencias y avances de todas las acciones en forma de transversalización de las humanidades como parte de la formación integral.

\section{Respuesta a la pregunta del programa de investigación}

Se parte de la pregunta de investigación: ¿cuál sería la formación en valoración integral de la enseñanza y aprendizaje que habría de proporcionarse a los docentes de la universidad para que éstos se conviertan en mediadores y promotores de competencias genéricas comunicativas de lectura crítica y construcción textual a través de las asignaturas -across disciplines- en los estudiantes? (Inciarte, García, Sánchez, Marín, Conde, Castillo y García-Martín, 2019). Para poder responder a la pregunta de investigación, conseguir los objetivos y contrastar las previsiones planteadas se proponen los siguientes dos tipos de estudios, de análisis de situación e instruccionales.

El primer tipo de estudios, en fase de culminación, de análisis de situación y necesidades percibidas (García et al., 2019). Estudios descriptivos, comparativos y de validación de instrumentos. Se ha desarrollado un análisis de detección de situación y necesidades con la intención de la inclusión de seguimientos, siguiendo la misma estrategia y lógica de los estudios sobre deserción estudiantil, permitiendo comparaciones por ciclos de año, tres años, etc (Fragozo-CUC1, 2009; Fragozo-CUC2, 2013). En esta primera fase se culminan estudios descriptivos del uso de SSEV y MMAC, comparativos con patrones psicológicos, pedagógicos, didácticos y educativos y resultados de aprendizaje en competencias genéricas, y de validación de instrumentos. Se han implementado dos tipos de instrumentos de Evaluación y Metodologías y su Incidencia en las Competencias Genéricas (EMICOG), una versión para profesores (García-Martín, García, Inciarte, Marín, Sánchez \& Conde, 2019a) y otra para estudiantes, con más medidas y variables medidas (García-Martín, García, Inciarte, Marín, Sánchez \& Conde, 2019b), incluyendo en ambos la detección de necesidades formativas.

El segundo tipo de estudios, se centra en la revisión, validación y contrastación de estrategias transversales exitosas. En una segunda fase, se culminarían estudios de búsqueda sistemática, de validación de actuaciones en desarrollo y, por último, estudios de contrastación de estrategias exitosas en contextos internacionales. Se trataría de identificar, analizar y contrastatar estrategias exitosas, validando las propias, e implementando otras de contextos internacionales, es decir, habría que hacer una búsqueda de estudios empíricos internacionales de introducción de la LC y la CT como actividades transversales significativas dentro de las asignaturas de los grados (pensar en implementarlas vía MOOCs u otras vías virtuales); habría que hacer estudios instruccionales o de intervención, tanto para validar las experiencias propias como para probar la eficacia y eficiencia de otras. Habría que diseñar un plan específico para estos estudios de la segunda fase con foco en actuaciones y estrategias directamente transversales sobre los alumnos a través del resto de materias y evaluar su eficacia y el papel mediador y modulador de las variables psicoeducativas introducidas en la primera fase (AE, IE, MOT, DS, AFR, ATR, CEAM/CEPEA/MSG...) y del uso del HEWE, además de medidas directas de las competencias genéricas (CCGG), a través de MOOCs u otras 


\section{ANTECEDENTES EXITOSOS PROPIOS - VALOR AÑADIDO PARA LA EDUCACIÓN SUPERIOR EN COLOMBIA}

actuaciones virtuales y semipresenciales (García-Martín \& García, 2017; García-Martín et al., 2015; 2016), utilizando diseños experimentales de instrucción comparativa (García-Martín \& García, 2018).

Igualmente, se prevé el análisis de aportaciones cualitativas de los diferentes sectores implicados, con el fin de implementar mejoras en base a las informaciones y datos objetivos y subjetivos recabados con el fin de que se trate de una investigación integrada en la propia mejora pedagógica y didáctica (cfr., Cunha et al., 2018). Las reflexiones teóricas y valorativas de los diferentes sectores y de los profesores investigadores del proyecto podrán ser objeto de alguna publicación específica, sobre todo para contrastar con las realizadas recientemente en Colombia (Arias-Gómez et al., 2018; Díaz-Flórez, 2018; Tamayo et al., 2018; OCDE, 2016) y a nivel internacional (Barnet, 2001; Ravitch, 2013). Este segundo tipo de estudios se implementarán durante los próximos tres años. Es preciso el diseño de varios grupos de instrucción con focos en sistemas de evaluación, competencias genéricas, metodologías docentes, fomento de estrategias de prácticas basadas en la evidencia de carácter innovador incluyendo evaluación de los resultados de aprendizaje (RRAA), niveles de logro académico, niveles de satisfacción de profesores y estudiantes; entre otros. Diferentes meta-análisis recientes así lo confirman (Miller et al., 2016; Peng et al., 2019; Scott et al., 2018; Uttl et al., 2917; Xie et al., 2018) así como los estudios empíricos recientes (Minghui et al., 2018; Widlund et al., 2018; Zeng et al., 2019).

\section{MODELO DE FOMENTO DE COMPETENCIAS GENÉRICAS EN TODOS LOS PROGRAMAS DE PRE- GRADO (UNIVERSIDAD DE LA COSTA)}

Para contextualizar el segundo tipo de estudios parece pertinente explicar el modelo concreto de actuación en relación con el fomento de las competencias, genéricas y específicas, a través de todas las asignaturas en todos los títulos de pregrado. En primer lugar, se parte del análisis hecho a los sistemas y procedimientos de evaluación existentes en la Institución y las concepciones de evaluación, implícitas y explícitas, en el marco de las innovaciones asumidas por La Universidad de la Costa CUC, a través de la Vicerrectoría Académica, desde donde se vienen liderando los procesos de formación por competencias, se propone un Modelo de Evaluación del Aprendizaje alineado a los modelos Pedagógico, Curricular y de Competencias ya implementados en la Institución, de modo que estos sean pertinentes y consistentes con las innovaciones curriculares implementadas, contribuyendo así a una mejor gestión de la calidad de los aprendizajes de los estudiantes y que se posibilite la toma de decisiones a partir de los resultados de la evaluación de los aprendizajes. Este proceso impacta a cada uno de los programas del Universidad.

El presente Modelo se constituye en documento orientador de la propuesta académica que lidera la evaluación del aprendizaje en la institución en sus niveles de pregrado; representa el fruto de un análisis detenido que ameritó la revisión del referente normativo a la luz del horizonte institucional en lo referente a Modelo Pedagógico, de Formación por Competencia y Curricular. La mirada fue discutida al interior de los distintos comités curriculares. En este sentido, pensar en el tipo de hombre que la sociedad demanda es una mirada que desde el quehacer de la Educación se debe atender. Para ello, existen aspectos fundamentales que regulan el sistema educativo en general y se normalizan desde puntos concretos a través de la ley, en el caso de Colombia su Ley 115 de 1994, Ley General de Educación que en su artículo 73 hace referencia a los Proyectos Educativos Institucionales. Y en la educación superior, por su parte, es reglamentada por la Ley 30 de 1992 que define el carácter y autonomía de las Instituciones de Educación Superior -IES-, que con sustento en el Ministerio de Educación Nacional y sirve de marco a las acciones que desde las Instituciones Educativas se emprenden. Por otra parte, existe el PEI, y en la dinámica de éste, el diseño curricular que es particular para cada Institución Educativa. 
En este sentido y queriendo motivar de forma articulada los esfuerzos institucionales en el desarrollo de las competencias, tanto específicas como genéricas, de los estudiantes; se vienen desarrollando, en la Institución, acciones que propenden por el fortalecimiento de las mismas. En la línea de lo anterior, se reconoce en el acuerdo, No. 638 del 26 de noviembre de 2014 el compromiso institucional con el fortalecimiento permanente de las competencias genéricas; entendidas desde la declaración dada en el modelo curricular como: "las capacidades y habilidades compartidas o comunes a cualquier campo de estudio", es decir, que las competencias en los perfiles de cualquier titulación son similares, dado que son genéricas en la educación superior o profesionalidad de las personas, dentro de las cuales se destacan la ética profesional, compromiso con la calidad, construcción textual, razonamiento cuantitativo, competencias ciudadanas, lectura crítica e inglés, de acuerdo con las directrices del Instituto Colombiano para la Evaluación de la Educación (ICFES - MEN, 2018).

La Universidad de la Costa, asume las competencias genéricas como un conjunto de funciones que se ejercen en cumplimiento del desempeño laboral, independiente de la profesión u ocupación. Son, entonces, competencias diversas a las disciplinares que complementan el quehacer disciplinar de las distintas profesiones de hoy en día, constituyéndose, el desarrollo de éstas, en parte integral de los perfiles profesionales de los programas académicos.

La estrategia consiste en que desde las asignaturas del plan de estudio se implementan actividades que incentiven el desarrollo de las competencias genéricas, tales como lectura crítica, comunicación escritura, razonamiento cuantitativo, competencias ciudadanas, e inglés. Estas competencias son evaluadas a través de una prueba estandarizada que desarrollan los estudiantes de la universidad de forma censal y que tiene una ponderación del 10\% de la calificación final. De esta forma el sistema de calificación de la universidad se ajusta a la siguiente distribución de tres evaluaciones en cada período semestral con un valor cada uno del $30 \%$ al que se le añade el $10 \%$ de resultados en la prueba de competencias genéricas (cfr., Figura 1).

Figura 1. Evaluación del aprendizaje en un semestre-tipo en cualquier asignatura de pregrado, con tres evaluaciones formativas que representan el $90 \%$, más el $10 \%$ de los resultados en las pruebas de competencias genéricas.

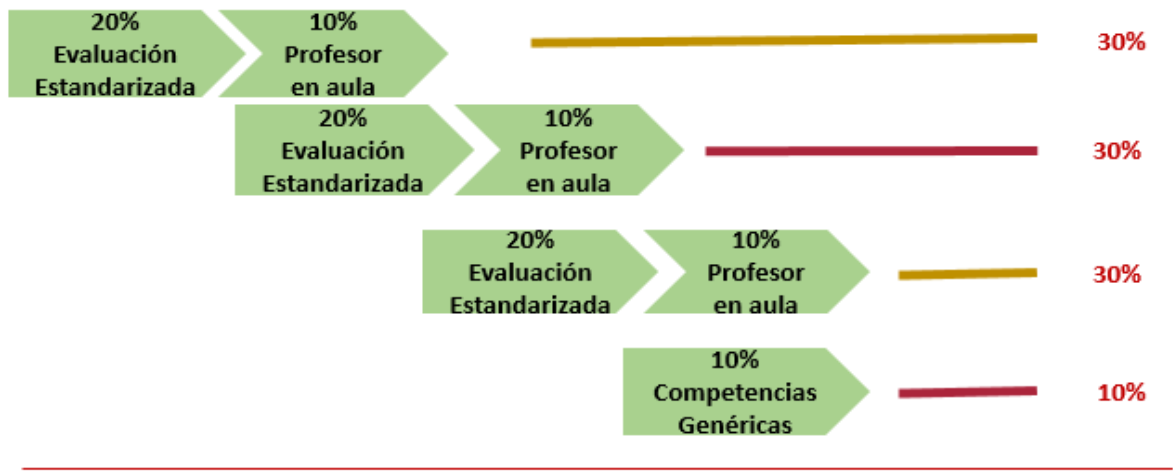

\section{Evaluación del Aprendizaje}

Dentro de las actividades que implementan los profesores en aula y que promueven el fortalecimiento de las competencias genéricas: los programas académicos a través de una ruta implementan en aula el desarrollo de las competencias. 


\section{ANTECEDENTES EXITOSOS PROPIOS - VALOR AÑADIDO PARA LA EDUCACIÓN SUPERIOR EN COLOMBIA}

En términos de resultados, se observan aspectos positivos que se destacan por la participación de los estudiantes en esta apuesta institucional, mostrando los siguientes resultados en los periodos académicos 2018-1 2018-2 (cfr., Figura 2).

Figura 2. Resultados en las pruebas de competencias genéricas durante el primer y segundo semestres de 2018.

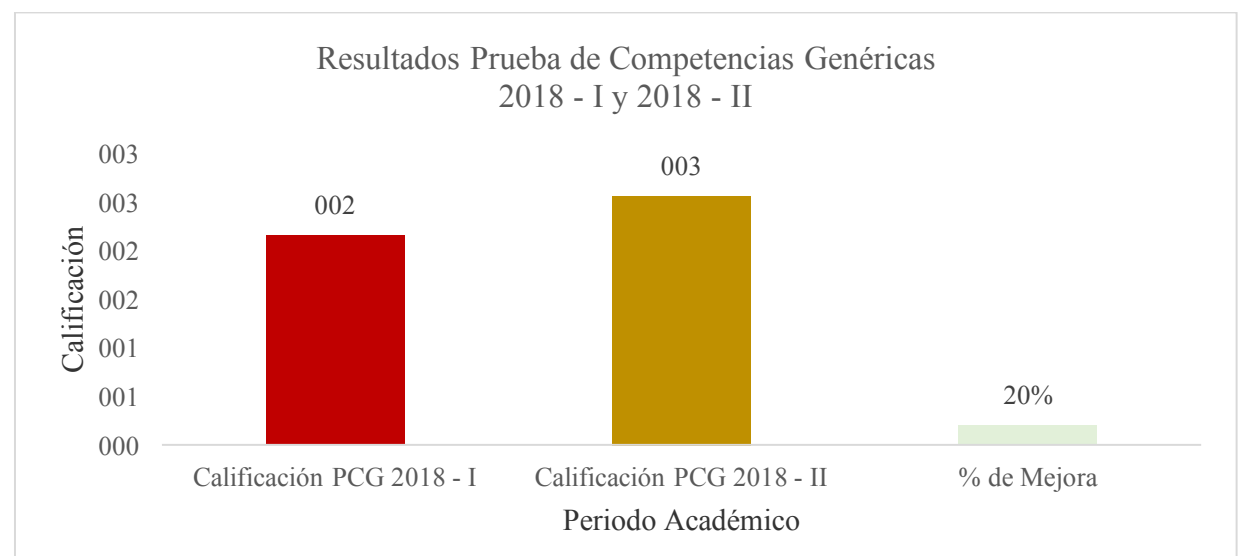

De igual forma se tienen resultados filtrados por competencia que permiten el análisis y la toma de decisiones de cara a su fortalecimiento, tal y como se muestra en la gráfica siguiente (véase Figura 3).

Figura 3. Resultados por tipo de competencia genérica durante los dos semestres de 2018.

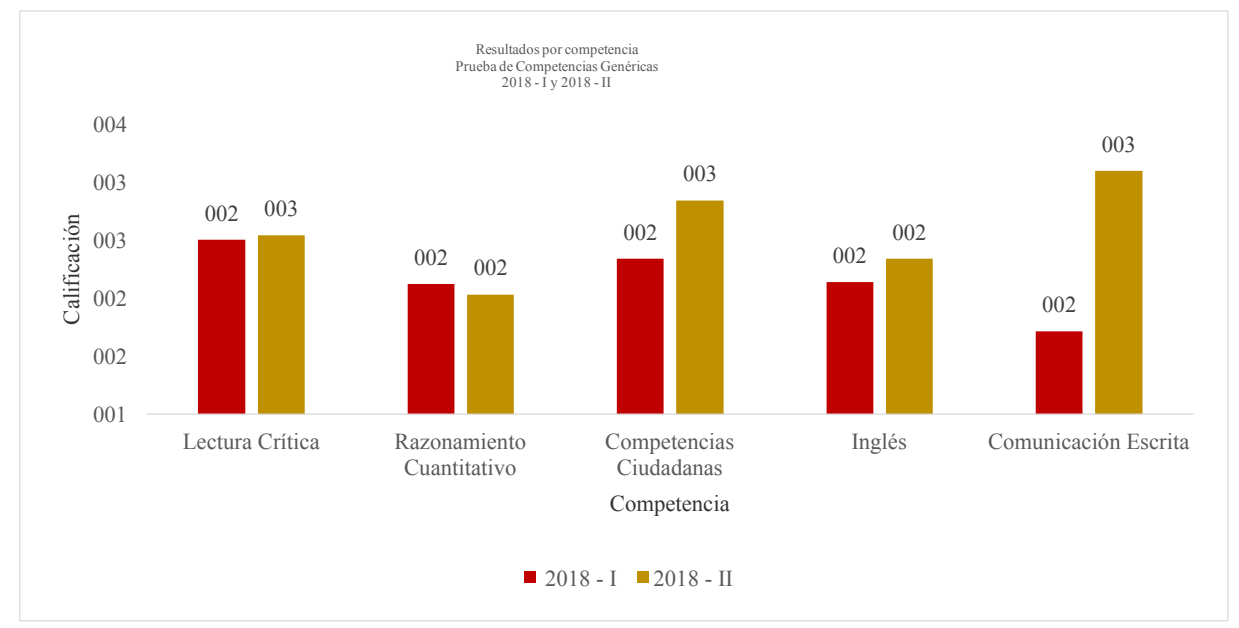

Los esfuerzos institucionales de cara al fortalecimiento de las competencias genéricas se constituyen en un desafío que compromete a todos los programas y que demanda de un periodo de madurez y apropiación por parte de los participantes institucionales. 
La Universidad de la Costa invierte en la formación de los miembros de la comunidad académica, generando capacidad instalada, que permita alcanzar la meta institucional planteada en referencia al desarrollo de las competencias genéricas en la institución.

\section{DISCUSIÓN Y CONCLUSIONES}

La revisión de experiencias exitosas y variadas en la institución educativa superior de la Universidad de la Costa, ilustrado por el modelo de evaluación y enseñanza transversal de las competencias genéricas en todas las asignaturas, sintetizado en la estrategia "más diez por ciento", representa la justificación y el marco en donde se inscribe el actual programa de investigaciones Evaluación y Metodologías Docentes y su Incidencia en las Competencias Genéricas (EMICOG), estando concluyendo el primer tipo de estudios descriptivos y comparativos; y queda enmarcada con gran solidez y rigor, además de relevancia educativa y científica, la siguiente fase de validación de alguna de estas experiencias de transversalización de las competencias genéricas a través de todas las asignaturas de todos los programas de pregrado de la Universidad de la Costa.

En conclusión, sea en base a los antecedentes del contexto propio, sea en base a los antecedentes internacionales, la justificación del presente estudio parece evidente. Se precisa, no solo estudiar la situación actual del uso real de sistemas de evaluación y metodologías activas por los profesores y su relación con patrones psicoeducativos y el papel que juegan en los resultados de aprendizaje y en las competencias genéricas y de necesidades formativas de los docentes (estudios descriptivos, estudios comparativos de patrones psicoeducativos y de validación de instrumentos) en una primera fase; sino que además es preciso la identificación y el análisis de estrategias eficaces directamente aplicables de forma transversal, por lo que las tendencias indicadas pueden mostrar posibles vías de actuación (estudios de validación de prácticas exitosas implementadas en la CUC, estudios instruccionales, estudios de formación de docentes, estudios de implementación vía M00Cs u otros), para las siguientes fases del programa de investigación (García-Martín \& García, 2018; García-Martín, García, Álvarez \& Caso, 2015).

Entre el impacto del programa de investigación, aparte del científico, el social, el ambiental, en la productividad y productos obtenidos, sea en forma de instrumentos de evaluación y detección de necesidades formativas (García-Martín et al., 2019a; 2019b; Inciarte et al., 2019), además de la necesidad de validar las propias experiencias, se espera aportar difusión entre la comunidad científica de avances de resultados descriptivos, comparativos, de validación de instrumentos, de revisión de antecedentes de estrategias exitosas internacionales en congresos internacionales como el actual; la producción de artículos científicos en foros de impacto internacional en open acess (ej., Frontiers in Psychology). Además, difusión entre la comunidad Unicosta y comunidad educativa nacional, con aportaciones a jornadas y seminarios de difusión de su implementación, desarrollo y resultados y el diseño de actividades transversales de promoción de competencias genéricas a través de las asignaturas de todos los pregrados, y sobre todo en lectura crítica y construcción textual, o con el diseño de cursos MOOCs y su implementación para el fomento de CCGG transversales autofinanciables y autorrealizables y programados ofertados a la comunidad educativa propia y externa (CEEDAR, 2019a; 2019b; Miller et al., 2016; Scott et al., 2018; USOE, 2019).

\section{REFERENCIAS BIBLIOGRÁFICAS}

Arias Gómez, D. H., Díaz Flórez, O. C., Garzón Barragán, I., León Palencia, A. C., Rodríguez Ávila, S. P., \& Valbuena Ussa, E. 0. (2018). Entre las exigencias de calidad y las condiciones de desigualdad: Formación inicial de profesores en Colombia. Bogotá: UPN

Barnet, R (2001). Los límites de la competencia. El conocimiento, la educación superior y la sociedad. Barcelona: Gedisa 


\section{ANTECEDENTES EXITOSOS PROPIOS - VALOR AÑADIDO PARA LA EDUCACIÓN SUPERIOR EN COLOMBIA}

Canedo-García, A., García-Sánchez J. N., \& Pacheco-Sanz D. I. (2017). A systematic review of the effectiveness of intergenerational programs. Frontiers in Psychology, 8(1882), 1-13. doi: 10.3389/fpsyg.2017.01882

CEEDAR (2019a). Evidences Based Practices. Ceedar innovation configurations. Tools for programs reforms. Retrieved from http://ceedar.education.ufl.edu/tools/innovation-configurations/

CEEDAR (2019b). The CEEDAR Center Evidence Standards. Retrieved from http://ceedar.education.ufl.edu/wp-content/uploads/2014/08/Evidence-Based-Practices-guide.pdf

Chen, Y. H., \& Lin, Y. J. (2018). Validation of the short self-regulation questionnaire for Taiwanese College Students (TSSRQ). Frontiers in Psychology, 9(259), 1-10. doi: 10.3389/fpsyg.2018.00259

CUC (2018). Autoinforme de Acreditación. Barranquilla: CUC

Cuesta M., Suárez-Álvarez J., Lozano L. M., García-Cueto E., \& Muñiz J. (2018). Assessment of eight entrepreneurial personality dimensions: validity evidence of the BEPE Battery. Frontiers in Psychology, 9(2352), 1-10. doi: 10.3389/fpsyg.2018.02352

Cunha J., Rosário P., Núñez J. C., Nunes A. R., Moreira, T., \& Nunes, T. (2018). "Homework Feedback Is. ...: Elementary and middle school teachers' conceptions of homework feedback. Frontiers in Psychology, 9(32), 1-20. doi: 10.3389/fpsyg.2018. 00032

Díaz, C., \& García, J. N. (2016a). Psychological profiles of older adult Web 2.0 tool users. Computers in Human Behavior, 64, 673-681. doi: 10.1016/j.chb.2016.07.007

Díaz, C., \& García, J. N. (2016b). Identification of relevant elements for promoting eficient interventions in older people. Revista de Psicodidáctica (English Edition), 21(1), 157-173. doi: 10.1387/RevPsicodidact.13854

Díaz-Flórez, 0. C. (2018). Las competencias en la educación superior. Debates contemporáneos. Tesis Doctoral. Bogotá: UPN

García-Martín, J., \& García-Sánchez (2013). Patterns of Web 2.0 tool use among young Spanish people Computers \& Education, 67, 105-120. doi: 10.1016/j.compedu.2013. 03.03.003

García-Martín, J., \& García-Sánchez, J. N. (2015). Use of Facebook, Tuenti, Twitter and Myspace among young Spanish people. Behaviour \& Information Technology, 34(7), 34-37. doi: 10.1080/0144929X.2014.993428

García-Martín, J., \& García-Sánchez, J. N. (2017). Pre-service teachers' perceptions of the service competence dimensions of digital literacy and of psychological and educational measures. Computers \& Education, 106, 1-14. doi: 10.1016/j.compedu. 2016.12.010

García-Martín, J., \& García-Sánchez, J. N. (2018). The instructional effectiveness of two virtual approaches: processes and product. Revista de Psicodidáctica (English Edition), 23(2), 117127. doi: 10.1016/j.psicod.2018.02 .002

García-Martín, J., García, J. N., Inciarte, A. J., Marín, F. V., Sánchez, E. R., \& Conde, M. E. (2019a). Sistemas de evaluación y metodologías docentes y su incidencia en las competencias genéricas (EMICOG - profesores). International Journal of Developmental and Educational Psychology, 31 (1)

García-Martín, J., García, J. N., Inciarte, A. J., Marín, F. V., Sánchez, E. R., \& Conde, M. E. (2019b). Sistemas de evaluación y metodologías docentes y su incidencia en las competencias genéricas (EMICOG - estudiantes). International Journal of Developmental and Educational Psychology, 31 (1)

García, J. N., Pacheco, D. I., Díaz, C., \& Canedo, A. (2016). Fidelidad de las prácticas basadas empíricamente en dificultades del desarrollo y del aprendizaje. En J. L. Castejón Costa (Coord.) Psicología y Educación: Presente y Futuro (pp. 75-91). Alicante: ACIPE. Retrieved from: 
http://www.cipe2016.com/cipe_fin al_capitulos.pdf

García, J. N. (Ed.) (2016). Issues in pedagogy. Research collection. Rijeka: InTech. 112 pp. (ISBN: 978-953-51-2338-5)

García, J. N., Conde, M. E., Inciarte, A. J., Sánchez, E. R., Marín. F. V., \& García-Martín, J. (2019). Revisión de estudios internacionales sobre evaluación y metodologías docentes universitarias. International Journal of Developmental and Educational Psychology, 31(1)

Habók, A., \& Magyar, A. (2018). Validation of a self-regulated foreign language learning strategy questionnaire through multidimensional modelling. Frontiers in Psychology, 9, 1-11. doi: 10.3389/fpsyg.2018.01388

Inciarte, A. J., García, J. N., Sánchez, E. R., Conde, M. E., Marín. F. V., Castillo, J. S., y García-Martín, J. (2019). Incidencia de la evaluación y las metodologías docentes en el desarrollo de competencias genéricas. International Journal of Developmental and Educational Psychology, 31(1)

ICFES-MEN (2018): Guía de Orientación Saber Pro. Módulo de Competencias Genéricas 2018. Bogotá: Instituto Colombiano para la Evaluación de la Educación ICFES Ministerio de Educación Nacional MEN. Recuperado de http://www.icfes.gov.co/ documents/20143/496194/Guia\%20de\%20orientacion\%20modulos\%20de\%20competencias\%20genericas-saber-pro-2018.pdf

Li, J., Ye, H., Tang, Y., Zhou, Z., \& Hu, X. (2018). What are the effects of self-regulation phases and strategies for Chinese students? A meta-analysis of two decades research of the association between self-regulation and academic performance. Frontiers in Psychology, 9, 1-13. doi: 10.3389/fpsyg.2018.02434

Martínez-Ferrer, B., Moreno, D., \& Musitu, G. (2018). Are adolescents engaged in the problematic use of social networking sites more involved in peer aggression and victimization? Frontiers in Psychology, 9(801), 1-13. doi: 10.3389/fpsyg.2018.00801

Miller, D. M., Scott, C. E., \& McTigue, E. M. (2016). Writing in the secondary-level disciplines: A systematic review of context, cognition and content. Educational Psychology Review, 30(1), 83120. doi: $10.1007 / \mathrm{s} 10648-016-9393-z$

Minghui, L., Lei, H., Xiaomeng, C., \& Potmešilc, M. (2018). Teacher efficacy, work engagement, and social support among Chinese special education school teachers. Frontiers in Psychology, 9(648), 1-8. doi: 10.3389/fpsyg.2018.00648

OCDE. (2016). La educación en Colombia. MEC: OCDE. https://www.mineducacion. gov.co/1759/articles-356787_recurso_1.pdf

Oriol, X., Miranda, R., Oyanedel, J.C., \& Torres, J. (2017). The role of self-control and grit in domains of school success in students of Primary and Secondary School. Frontiers in Psychology, 8(1716), 1-9. doi: 10.3389/fpsyg.2017.01716

Peng, P., Wang, T., Wang, C., \& Lin, X. (2019). A Meta-analysis on the relation between fluid intelligence and reading/mathematics: effects of tasks, age, and social economics status. Psychological Bulletin, 145(2),189-236. doi: 10.1037/bul0000182

Puente-Martínez, A., Páez, D., Ubillos-Landa, S., \& Da Costa-Dutra, S. (2018). Examining the structure of negative affect regulation and its association with hedonic and psychological wellbeing. Frontiers in Psychology, 9(1592), 1-14. doi: 10.3389/fpsyg. 2018.01592

Ravitch, D. (2013). Reign of Error: The Hoax of the Privatization Movement and the Danger to America's Public Schools. New York: Alfred A. Knopf.

Robledo, P., \& García, J. N. (2018). Description and Analysis of Strategy-Focused Instructional Models for Writing. En R. Fidalgo y T. Olive (Series Eds.) y R. Fidalgo, K.R. Harris, y M. Braaksma, (Vol Eds.), Studies in Writing Series: Vol. 34. Design Principles for Teaching Effective 


\section{ANTECEDENTES EXITOSOS PROPIOS - VALOR AÑADIDO PARA LA EDUCACIÓN SUPERIOR EN COLOMBIA}

Writing, (pp. 38-65). Leiden: Brill.

Robledo, P., García, J. N., Díez, C., Álvarez, M. L., Marbán, J. M., de Caso, A. M., Fidalgo, R., AriasGundín, O., \& Pacheco, D. I. (2010). Estilos de pensamiento y aprendizaje en estudiantes de magisterio y psicopedagogía: Diferencias según curso y especialidad. Escritos de Psicología, 3(3), 27-36. doi: 10.5231/psy.writ.2010.0707.

Romera, E. M., Herrera, M., Casas, J. A., Ortega, R., \& del Rey, R. (2018). How much do adolescents cybergossip? Scale Development and Validation in Spain and Colombia. Frontiers in Psychology, 9(126), 1-10. doi: 10.3389/fpsyg.2018.00126

Scott, C. E., McTigue, E. M., Miller, D. M., \& Washburn, E. K. (2018). The what, when, and how of preservice teachers and literacy across the disciplines: A systematic literature review of nearly 50 years of research. Teaching and Teacher Education 73, 1-13. doi: 10.1016/j.tate.2018.03.010

Sinval, J., Marques-Pinto, A., Queirós, C., \& Marôco, J. (2018). Work engagement among rescue workers: psychometric properties of the Portuguese UWES. Frontiers in Psychology, 8(2229), 1-16. doi: 10.3389/fpsyg.2017.02229

Tamayo Valencia, L. A., Niño Zafra, L. S., Cardozo Espitia, L. S., \& Bejarano Bejarano, 0. (2018). ¿Hacia dónde va la evaluación? Aportes conceptuales para pensar y transformar las prácticas de evaluación. Bogotá: IDEP

USOE (2019). OSEP Ideas that Work. Office of Special Education Programs, U.S. Department of Education. Retrieved from https://www.osepideasthatwork.org/

Uttl, B., White, C. A., \& Wong Glez, D. (2017). Meta-analysis of faculty s teaching effectiveness: Student evaluation of teaching ratings and student learning are not related. Studies in Educational Evaluation, 54, 22-42. doi: 10.1016/j.stueduc.2016. 08.007

Widlund, A, Tuominen, H., \& Korhonen, J. (2018). Academic well-being, mathematics performance, and educational aspirations in lower secondary education: changes within a school year. Frontiers in Psychology, 9(297), 1-20. doi: 10.3389/ fpsyg.2018. 00297

Xie, C., Wang, M., \& Hu, H. (2018). Effects of Constructivist and Transmission Instructional Models on Mathematics Achievement in Mainland China: A Meta-Analysis. Frontiers in Psychology, 9(1923), 1-18. doi: 10.3389/fpsyg.2018.01923

Zeng, G., Chen, X., Cheung, H. Y., \& Peng, K. (2019) Teachers' Growth Mindset and Work Engagement in the Chinese Educational Context: Well-Being and Perseverance of Effort as Mediators. Frontiers in Psychology, 10(839), 1-10. doi: 10.3389/fpsyg.20 19.00839 